\title{
Timing relations in speech and the identification of voice-onset times: A stable perceptual boundary for voicing categories across speaking rates
}

\author{
VICTOR J. BOUCHER \\ Université de Montréal, Montréal, Québec, Canada
}

\begin{abstract}
This study shows that the ratio of voice onset time (VOT) to syllable duration for $/ \mathrm{t} /$ and $/ \mathrm{d} / \mathrm{presents}$ distributions with a stable boundary across speaking rates and that this boundary constitutes a perceptual criterion by which listeners judge the category affiliation of VOT. In Experiment 1, best-fit regression lines for VOT ratios of intervocalic/t/ and / $/$ / against speaking rate had zero slopes, and there was an inferable boundary between the distributions. In Experiment 2, listeners' identifications of syllableinitial stops conformed to this boundary ratio. In Experiment 3, VOT was held constant, while VOT ratios were altered by modifying the duration of the following vowel. As VOT ratios exceeded the boundary estimated from the data of Experiment 1, listeners' identifications shifted from $/ \mathrm{d} /$ to $/ \mathrm{t} /$. Timing relations in speech production can determine the identification of voicing categoriesacross speaking rates.
\end{abstract}

Temporal aspects of speech such as voice onset time (VOT) have been used extensively in research on speech perception. VOT as an acoustic cue of voicing distinctions (Abramson \& Lisker, 1970) has been applied in studies on neural feature detectors, in clinical protocols, and in research on the perception of newborns and lower order mammals (e.g., Clarkson, Eimas, \& Marean, 1989; Eimas, Miller, \& Jusczyk, 1987; Kuhl \& Miller, 1978; Sinex \& Narayan, 1994; Stark \& Tallal, 1988). In all these instances, measurement and manipulation of voice onsets have been performed through reference to absolute numbers of milliseconds of VOT. However, studies have shown that such measures of VOT do not specify perceived voicing categories unambiguously (Green, Stevens, \& Kuhl, 1994; Kidd, 1989; Miller, Aibel, \& Green, 1984; Summerfield, 1975, 1981). This result is especially clear if one considers the effects of speaking rate. For instance, as speaking rate increases, the VOTs of voiceless stops shorten substantially and become similar to those of voiced stops spoken at slower rates (e.g., Miller \& Volaitis, 1989). Listeners can nonetheless consistently identify the heard onsets as belonging to voiced or voiceless categories. Reports also indicate that such effects are not limited to VOT but extend to other temporal properties relating to features of place and manner of articulation (Miller \& Baer, 1983; Miller \& Liberman, 1979).

I thank Quentin Summerfield, John Kingston, and Randy Diehl for their insights and numerous comments on previous versions of this paper. Correspondence concerning this article should be addressed to V. J. Boucher, Laboratoire des sciences phonétiques, Département de linguistique et de traduction, Université de Montréal, C. P. 6128 succursale Centre-ville, Montréal, QC, H3C 3J7 Canada (e-mail: victor.boucher@ umontreal.ca).
That listeners can identify variable VOTs in terms of given phonemic categories has been explained through reference to two general hypotheses characterized as $e x$ trinsic and intrinsic views of timing perception (Summerfield, 1981). The extrinsic account suggests that listeners adjust a criterion value used to make the voicing decision according to information bearing on speaking rate (e.g., Diehl \& Walsh, 1989; Dupoux \& Green, 1997; Pind, 1995; Pisoni, Carrell, \& Gans, 1983; Wayland, Miller, \& Volaitis, 1994). Because the VOTs for voiceless stops tend to shorten at fast rates of speech, listeners adjust the perceptual boundary toward shorter values. An intrinsic timing hypothesis, on the other hand, suggests that listeners rely on local timing relations, which can be measured as durational ratios (such as VOT/syllable duration). Such ratios are seen to be "self-normalizing" and intrinsically specify the category affiliation of heard VOTs in that "rate does not mediate the interpretation of the events" (Summerfield, 1981,p. 1091).

The views above differ fundamentally on the timing metrics that are used to measure speech and on assumptions of the origin or basis of the perceptual boundary. The latter notion is central in evaluating the hypotheses above in their capacity to predict listeners' identifications. An extrinsic timing account assumes that, with changes in speaking rate, there is a shift in the perceptual boundary. In previous reports, researchers have attempted to estimate the boundary shift not only for VOT but also for other timing attributes. For instance, by comparing production and perception data on the duration of formant transitions for $/ b /$ and /w/, Miller and Baer (1983) estimated that a perceptual criterion was located midway between two best-fit regression lines representing the formant changes of $/ \mathrm{b} /$ and $/ \mathrm{w} /$. Another approximation, which roughly amounted to a mid- 

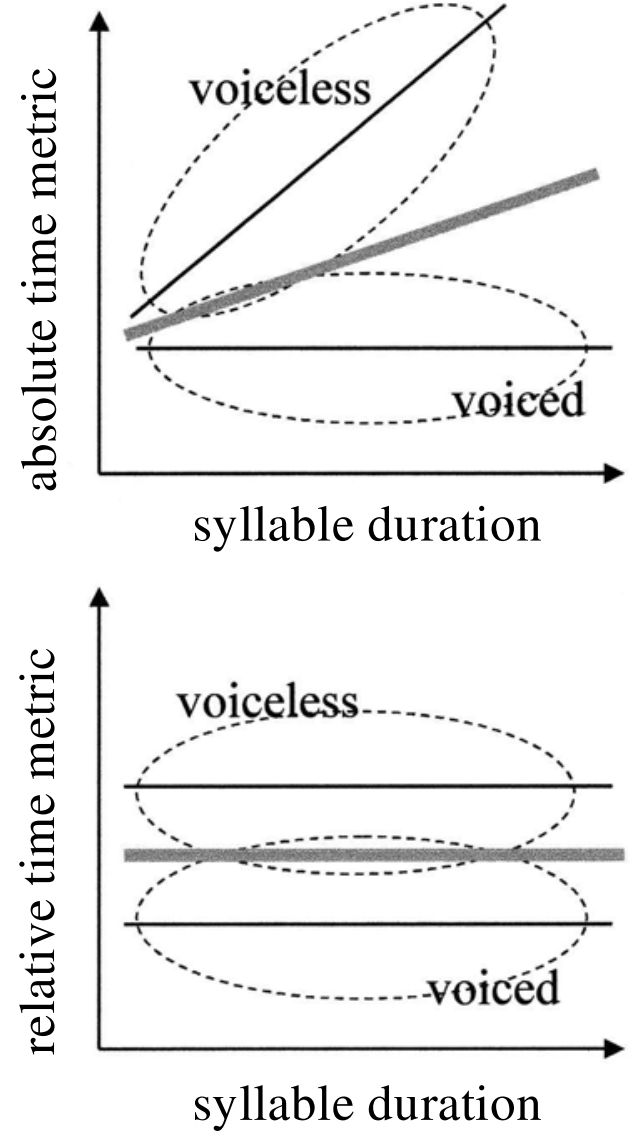

Figure 1. Hypothetical distributions of VOTs for voiced and voiceless stops. The top panel illustrates distributions for absolute measures of VOT. The wide bar indicates a midpoint boundary between two best-fit regression lines (shown by narrow lines). As suggested in an extrinsic timing hypothesis, this boundary shifts upward with a decrease in speaking rate. The bottom panel illustrates that, in terms of durational ratios of VOT, a stable boundary may occur across changes in speech rate.

point division, was proposed by Miller, Green, and Reeves (1986) for VOTs in /pi/and/bi/. These proposals provided an approximation of a changing perceptual boundary of the type illustrated in the top panel of Figure 1, where a division line midway between two distributions of values can be seen to shift upward as speaking rate decreases or as syllable durations increase. With respect to VOT, Miller et al. (1986) remarked that an optimal boundary estimated from their production data did not correspond to the criteria values reported in earlier work on the perception of voice onsets. In a further study, Miller and Volaitis (1989) attempted to assess the location of the $/ \mathrm{p} /-/ \mathrm{b} /$ boundary for VOT by using an identification task with two syllable durations of 125 and $325 \mathrm{msec}$. The identification function for a [pi]-[bi] continuum revealed a boundary at $35.6 \mathrm{msec}$ of VOT for a syllable duration of $125 \mathrm{msec}$, which did not correspond to the criterion of $14-15 \mathrm{msec}$ obtained in the production data for syllable durations of
100-199 msec (Miller et al. 1986). At the longer syllable duration of $325 \mathrm{msec}$, Miller and Volaitis noted that listeners' identificationssuggested $\mathrm{a} / \mathrm{p} / \mathrm{-} / \mathrm{b} /$ boundary at a VOT of $43.9 \mathrm{msec}$, which was above the boundary of $27-28 \mathrm{msec}$ reported by Miller et al. (1986) for syllables ranging from 300 to $349 \mathrm{msec}$. These results indicated that a shifting boundary between voiced and voiceless VOTs estimated from speech production data did not serve to predict listeners' identifications of voicing categories across speaking rates.

As for an intrinsic timing hypothesis, research has not established a criterion ratio for VOT that would allow an evaluation of this hypothesis in predicting changes in an identification function. A recent study by Kessinger and Blumstein (1998) suggests that ratios of VOT to syllable duration for voiceless stops in repeated nonwords manifest a limited degree of variability across speaking rates. Such findings are compatible with several reports of stable timing relations in speech production (Harris, Tuller, \& Kelso, 1986; Nittrouer, Munhall, Kelso, Tuller, \& Harris, 1988; Tuller \& Kelso, 1984). Kelso, Saltzman, and Tuller (1986) have shown that the timing relations between oral and glottal motions, which are related to ratios of VOT (Tuller \& Kelso, 1991), can remain stable across a range of speaking rates. Limited variability in the production of VOT ratios may specify a stable perceptual boundary that is parallel to the rate axis, as is illustrated in the bottom panel of Figure 1. This leads to a different set of predictions with respect to extrinsic timing accounts.

One prediction can be related to the observation that absolute VOTs for voiced and voiceless consonants tend to converge at fast speaking rates. If listeners are attending to the absolute times of voice onsets, the accuracy of their identifications should decrease for VOTs at fast rates of speech, where values are seen to approach the assumed boundary point. The assumption that listeners are relying on durational ratios of VOT suggests a different prediction. The hypothesis of a boundary and distributions that are parallel to the rate axis suggests that the accuracy of listeners' judgments may not vary substantially with changes in speaking rate (see Figure 1). Defining a criterion ratio for voiced and voiceless VOTs can also serve to devise critical experiments. If listeners rely on durational ratios, a modification of the duration of neighboring elements that would alter the ratio of VOT (but not its absolute duration) should lead to a change in the identification function. If one assumes that the perceptual boundary is specified by timing relations in speech, the point at which a category change occurs in listeners' identifications should correspond to the boundary observed in the production of VOT ratios for voiced and voiceless consonants.

This conception of the role of timing relations departs from an earlier class of intrinsic timing theories that have been seen to rest crucially on the assumption of constant ratios in speech (e.g., Fowler, 1980; Kelso \& Tuller, 1985). Several authors have categorically rejected temporal ratios in production as having any role in speech perception, on the grounds that such measures fail to reveal invariant 
attributes that would allow a direct mapping of phonemic features (Keller, 1987, 1989; Löfqvist, 1991; Luce \& Charles-Luce, 1985; Pisoni, 1986). For example, some authors see that an intrinsic timing view posits that VOT and syllable duration change with speaking rate "in such a way that this functional relation, or higher-order property, remains invariant" (Wayland et al., 1994, p. 2699). Because of the failure to find temporal constants in speech, relativetiming hypotheses have been largely dismissed (see the comments of Pind, 1995). There is substantial evidence that timing relations involving such aspects as VOT or formant transitions are not constant (e.g., Kessinger \& Blumstein, 1998; Port, 1981; Volaitis \& Miller, 1992). However, such observations do not provide incontestable grounds for rejecting intrinsic timing explanations. A temporal property of speech may vary substantially but still present distributions with stable boundaries that can determine the perception of phonemic categories.

In a recent report, Utman (1998) concluded that intrinsic timing may not explain listeners' identifications of VOT. The study involved stimuli constructed by cross-splicing VOTs of oral stops and vowels produced at slow and fast rates of speech, giving combinations such as a long voiceless VOT with a short vowel or a short voiceless VOT with a long vowel. Since listeners consistently identified the voiceless stop irrespective of variations in vowel length, it was seen that timing relations do not affect listeners' responses. The author did not report on whether or not the stimuli contained durational ratios similar to those of voiced stops in natural speech, and the use of stimuli with voiceless VOTs throughout may well have created large ratios that precluded the identification of voiced consonants. Utman's results also contrast with numerous studies showing that vowel length in synthesized and natural speech syllables influences listeners' classification of the voicing category of syllable-initial stops (e.g., Green et al., 1994; Port \& Dalby, 1982; Sawusch \& Newman, 2000). However, none of these studies addressed the issue of whether or not a criterion ratio estimated from speech production data serves to predict listeners' identifications of voicing categories.

The present study addresses two questions bearing on an intrinsic account of timing perception: (1) Do relative measures of VOT (VOT/syllable duration) for voiced and voiceless stops present distributions with a stable boundary across speaking rates, and (2) does this boundary correspond to a perceptual criterion by means of which listeners identify the category affiliation of VOTs? With respect to these questions, the viability of an intrinsic timing hypothesis was examined in three related experiments. The first experiment was aimed at determining whether a stable boundary can be inferred from the distributions of VOT ratios for voiced and voiceless stops produced at varying speech rates, as envisaged in the lower panel of Figure 1 . The working hypothesis was that, if the distributions supported a stable boundary, then best-fit regression lines for the VOT ratios of voiced and voiceless stops would have no common intercept and slopes approaching zero with respect to changes in speech rate. Given that this was founded to be the case, a unique criterion ratio could then be estimated. The purpose of the second experiment was to determine whether listeners' identifications of stops in syllables spoken at varying rates would reflect voicing decisions based on a stable boundary ratio. In the third experiment, the ratio of VOT for a voiced stop was manipulated by modifying the length of the following vowel in order to evaluate the applicability of the boundary criterion in predicting changes in listeners' identifications. Taken together, the experiments enabled an evaluation of whether or not the perceptual boundary by which listeners judge the category affiliation of VOT can be linked directly to timing relations in speech production.

\section{EXPERIMENT 1}

\section{Method}

Subjects and Procedure. Observations of the timing of voice onsets were obtained by measuring utterances produced by 7 monolingual speakers of American English. These individuals were male, ranging in age from 18 to 29 years, with no declared history of speech or hearing problems. The utterances consisted of several repetitions of $[\Lambda$ 'dæp] and [ $\Lambda$ 'tæp], which included intervocalic stops with voiceless closures so as to facilitate VOT measures and the splicing of syllables (in Experiments 2 and 3). It was assumed that the possible word boundary effects for such contexts would not be a significant source of variation, given that a previous study showed that the temporal attributes of /' $\mathrm{d} / \mathrm{V}$ and /' $\mathrm{t} / \mathrm{V}$ are similar in wordinternal and word-initial positions (Boucher, 1988). The subject sat in a sound-treated room with a lavalier condenser microphone (Audio-Technica, Model AT831b) placed on a boom at about $20 \mathrm{~cm}$ from the lips. The subject was asked to produce six successive repetitions of $[\Lambda$ 'tæp $]$ at a moderately slow rate (a spoken example was provided), pause, and then produce the same utterance with just a slight increase in speaking rate. Ten utterances were requested with instructions to produce the final (10th) utterance at a maximal rate. Given that this task required some familiarity with what was meant by a "slight increase in speaking rate," practice runs were provided in which instructions aimed at obtaining small increases in speech rate were given during the pauses. These practice runs continued until the subject achieved, without instruction, what was perceived to be progressive changes in speaking rate for the 10 utterances. Only the final series of 10 utterances produced by the speaker was retained for the analysis. The same procedure was used to solicit productions of the context [ $\Lambda^{\prime}$ dæp]. Measures were performed on a total 140 utterances recorded on digital audiotape (3M) using a TEAC recorder (Model DA-P20).

Measurements. The recorded signals were digitized at $10 \mathrm{kHz}$ and analyzed using CSL (Model 4300, Kay Elemetrics). With this system, signals are preemphasized at $6 \mathrm{~dB} /$ octave, and low-pass filtered at $40 \%$ of the sampling frequency, and a 16-bit A/D conversion provides a quantization at $64 \mathrm{~K}$ levels. VOT was measured by referring to scale-magnified oscillograms with synchronized spectrograms used as a general guide in locating vowel formants and consonant closure gaps (Brown, Morris, \& Weiss, 1993). In the contexts [ $\left.\Lambda^{\prime} \mathrm{dæp}\right]$ or [ $\Lambda$ 'tæp] involving the sequence of segments V1C1V2C2, VOT was measured as the time between the onset of the release burst of $\mathrm{C} 1$ and the onset of periodic energy (negative values in such measures indicate that onsets occur prior to the release). Ratios of VOT were calculated by referring to the duration of the C1V2 syllable delimited from the beginning of the closure interval for $\mathrm{C} 1(/ \mathrm{d} /$ or $/ \mathrm{t} /)$ to 


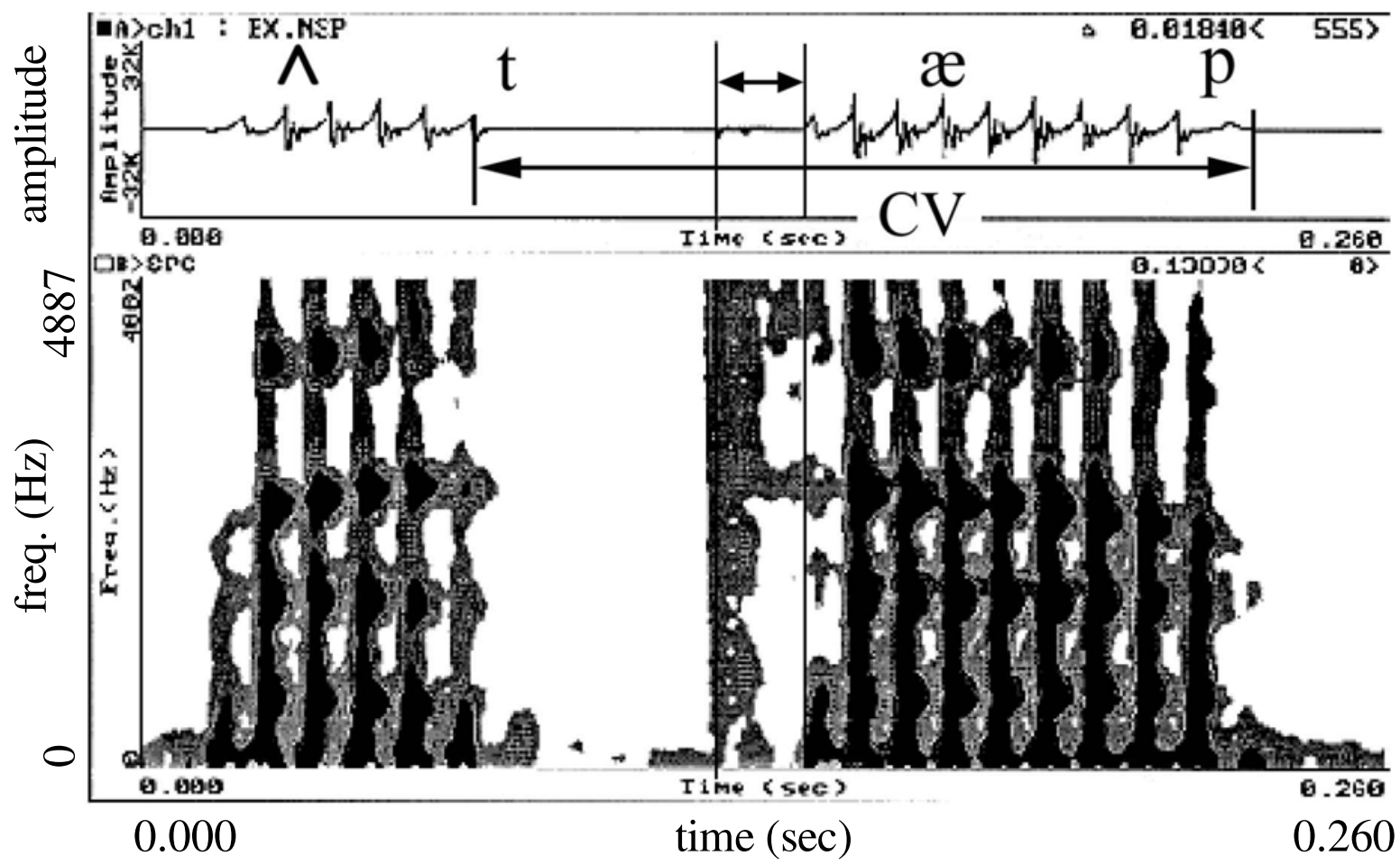

Figure 2. Example of a VOT measure for a voiceless stop and a CV segmentation.

the end of the periodic energy associated with V2. Figure 2 provides an example of the segmentation. Secondary analyses involved ratios calculated by referring to $\mathrm{V} 2 \mathrm{C} 2$ (delineated from the release burst prior to V2 to the end of the quasisilent gap of C2) and C1V2C2 (delimited from beginning of the gap for $\mathrm{C} 1$ to the end of the gap for $\mathrm{C} 2$ ).

Measurements were performed on three consecutive occurrences of $/ \mathrm{d} /$ or $/ \mathrm{t} /$ near the beginning of each utterance. The onsets of lowamplitude periodic energy were often difficult to delineate in certain cases, and for this reason all measurements of VOT were repeated by a separate judge. The results showed that VOT could not be established reliably, especially at fast rates of speech when voicing was either continuous or had varying negative values across the stop closure. VOT measures were also unreliable in some less frequent instances where V1 $([\Lambda])$ was either partly or entirely devoiced, which created geminate-like sequences (heard as $\left[\mathrm{p}^{\mathrm{h}} \mathrm{t}\right]$ or $\left[\mathrm{p}^{\mathrm{h}} \mathrm{d}\right]$ ). These cases were eliminated as were instances where interjudge measures of VOT exceeded $4 \mathrm{msec}$. This left a total of 164 values for $/ \mathrm{d} /$ and $188 \mathrm{for} / \mathrm{t} /$. Finally, one outlier, a /d/ segment produced as a heard aspirated $\left[\mathrm{t}^{\mathrm{h}}\right]$, was removed $(z=6.18, p<.001)$.

\section{Results}

The top panel of Figure 3 presents the VOTs of $/ \mathrm{t} /$ and /d/ observed for CV syllable durations varying from 124 to $429 \mathrm{msec}$. The best-fit regression lines for the aggregate data conform to previous observations showing substantial decreases in VOT for voiceless stops at fast rates of speech. That is, for /t/ VOT, Lane, Wozniak, Matthies, Svirsky, and Perkell (1995, note 1) calculated a regression slope of .14 from the data of Port and Rotunno (1979) and a slope of .17 from the data of Pind (1995) for Icelandic. These estimates approach the .15 slope observed in the present data (on the method used to calculate slopes and intercepts, see Kleinbaum, Kupper, \& Muller, 1988).

The bottom panel of Figure 3 shows the durational ratios of VOT (VOT/syllable duration). The suggestion that a stable boundary may be inferred from durational ratios of VOT implies, as stated in the working hypothesis, that best-fit regression lines for $/ \mathrm{t} /$ and $/ \mathrm{d} /$ ratios present nearzero slopes with respect to rate changes and share no common intercept. Regression lines for $/ \mathrm{t} /$ and $/ \mathrm{d} /$ values were obtained for each subject in order to compare the slopes and intercepts by means of paired $t$ tests. For the ratio measures, there were significant differences in the intercepts for the $/ \mathrm{t} /$ and $/ \mathrm{d} /$ regression lines $[t(6)=4.63, p=$ $.004]$, but no significant differences in the slopes $[t(6)=$ $1.00, p=.356]$. Furthermore, the slopes $\left(\beta_{1}\right)$ for all subjects clearly approached zero for $/ \mathrm{t} /$ and $/ \mathrm{d} /$ (i.e., for $/ \mathrm{t} /$, $3.2 \times 10^{-4}<\beta_{1}<1.7 \times 10^{-4}$; and for $/ \mathrm{d} /, 2.2 \times 10^{-4}<$ $\beta_{1}<.4 \times 10^{-4}$ ). These results contrast with those obtained with the use of absolute measures of VOT. In this case, no significant difference appeared between the / $t /$ and $/ \mathrm{d} /$ intercepts $[t(6)=1.21, p=.272]$, whereas significant differences were present for the slopes of absolute VOTs for $/ \mathrm{t} /$ and $/ \mathrm{d} /[t(6)=9.11, p<.001]$.

The findings confirm that a relative metric serves to reveal distributions of VOT ratios with best-fit regression lines that are parallel to the speech rate axis. This suggests that voiced stops tend to be characterized by a different value of the ratio of VOT to syllable duration from voiceless stops and that neither value varies with rate. Sec- 

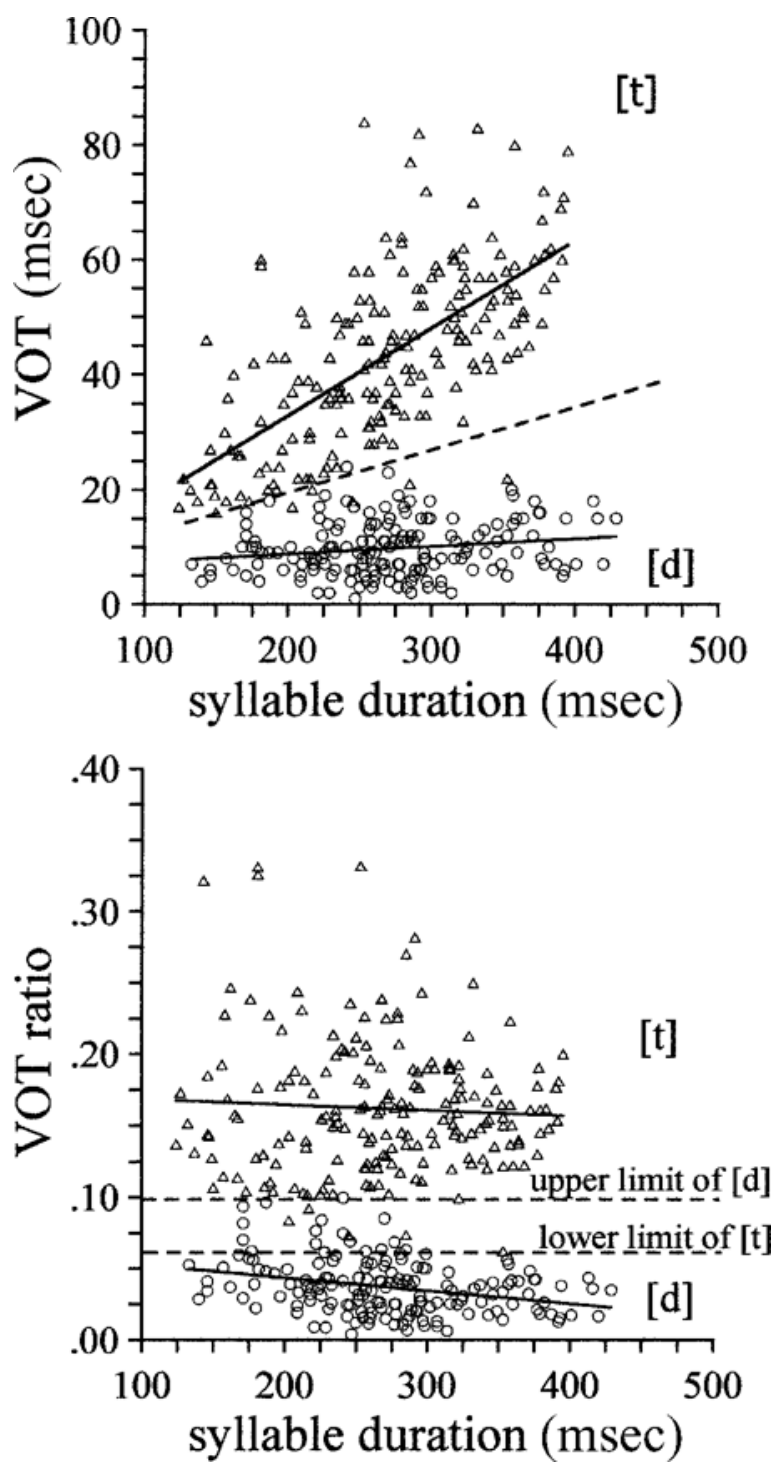

Figure 3. VOTs for $/ \mathrm{d} /$ and $/ \mathrm{t} / \mathrm{in}$ milliseconds (top panel) and corresponding ratios of VOT/syllable duration (bottom panel). Best-fit regression lines for the absolute VOTs present slopes of .152 for $/ \mathrm{t} /$ and .013 for $/ \mathrm{d} /$, with intercepts of $\mathbf{2 . 5 1 4} \mathrm{for} / \mathrm{t} /$ and 6.090 for $/ \mathrm{d} /$. Regression lines for the VOT ratios have slopes of .000 for both $/ t /$ and $/ d$, with intercepts of .173 for $/ t /$ and .062 for $/ \mathrm{d} /$. Note that the dotted line in the top panel represents an assumed boundary roughly midway between the two regression lines (see Miller et al., 1986). Dotted lines in the bottom panel represent a boundary region by reference to the minimum ratio produced for $/ \mathrm{t} /$ and the maximum ratio produced for $/ \mathrm{d} /$.

ondary analyses showed similar horizontal regression lines for $/ \mathrm{t} /$ and $/ \mathrm{d} /$ VOT ratios calculated by reference to $\mathrm{VC}$ and CVC syllables (as defined). Given these parallel regression lines, one may infer a stable boundary between $/ \mathrm{d} /$ and $/ \mathrm{t} /$ ratios across speaking rates. The location of the boundary was established with reference to the observed maximum VOT ratio of .099 that was produced for $/ \mathrm{d} /$ and the minimum ratio of .062 produced for $/ t /$. These values can be seen to define a general boundary region, as represented by the dotted lines in the bottom panel of Figure 3 . The following experiments were aimed at investigating whether such limits observed in the production of ratios of VOT correspond to a perceptual boundary.

It may be argued that a boundary may also be inferred between the absolute VOTs of / $\mathrm{t} /$ and /d/. Given previous suggestions (Miller \& Baer, 1983; Miller et al., 1986), one may estimate a boundary in terms of criteria values midway between the regression lines for $/ \mathrm{t} /$ and $/ \mathrm{d} /$ VOTs, as represented in Figure 3. Thus, one could contend that listeners' identifications of voicing categories are based on a shifting boundary rather than on a stable ratio. Experiments 2 and 3 served to assess these different hypotheses in accounting for listeners' identifications and in predicting category shifts in an identification function.

\section{EXPERIMENT 2}

\section{Method}

The aim was to establish whether listeners' identifications of voiced and voiceless stops in syllables produced at different rates of speech are compatible with the idea that the perception of voicing categories is determined by durational ratios of VOT or by the duration of VOT. The best-fit regression lines in the panels of Figure 3 show that, in terms of absolute measures, the difference between $/ \mathrm{d} /$ and $/ \mathrm{t} / \mathrm{VOT}$ tends to increase as speaking rate decreases, whereas durational ratios of VOT do not manifest this tendency. For the present experiment, stimuli representing these variations in the proximity of $/ t /$ and /d/ values across speaking rates were selected from the utterances of Experiment 1. Figure 4 presents the absolute VOTs and the corresponding durational ratios of four sets of four syllables produced by different speakers. The syllables were selected so that the differences in VOT ratios for the /d/ and / $/$ / sets were roughly similar for two different speaking rates. The change in speech rate was specified by a difference in syllable durations, which were within the ranges of 100-200 msec ("fast rate" condition) or 300-400 msec ("slow rate" condition).

Figure 4 shows that the application of relative and absolute timing metrics reveals varying distributions for voiced and voiceless VOTs in terms of their proximity with respect to the assumed boundaries. This leads to varying predictions bearing on the accuracy of listeners' identifications. In terms of absolute measures (top panel of Figure 4), the stimuli contain /t/ and /d/ VOTs that approach the boundary at fast rates of speech. Assuming that listeners rely on the millisecond values of voice onsets, one would expect a lower accuracy in listeners' judgments for both $/ \mathrm{t} /$ and $/ \mathrm{d} /$ in the fast speech condition but not in the slow speech condition. The bottom panel of Figure 4 suggests a different prediction in that the durational ratios of /t/ VOT are all outside the boundary region, whereas some of the /d/ values are at or within the boundary zone, especially in the fast speech condition. Thus, if listeners refer to timing relations, one would expect that the accuracy of listeners' identifications would be high for / $t /$ in both speech rate conditions but that accuracy of judgments would be lower for the stimuli containing $/ \mathrm{d} /$.

Subjects. The judges were 10 monolingual speakers of English ranging in age from 23 to 39 years. These subjects had not participated in Experiment 1 and were selected following a standard audiometric evaluation conforming to ANSI (1986) guidelines. That is, all subjects presented normal hearing thresholds bilaterally for octave frequencies extending from 250 to $8000 \mathrm{~Hz}$ inclusive.

Stimuli and Procedure. Using the editing options of the system described in Experiment 1, the 16 syllables represented in Figure 4 

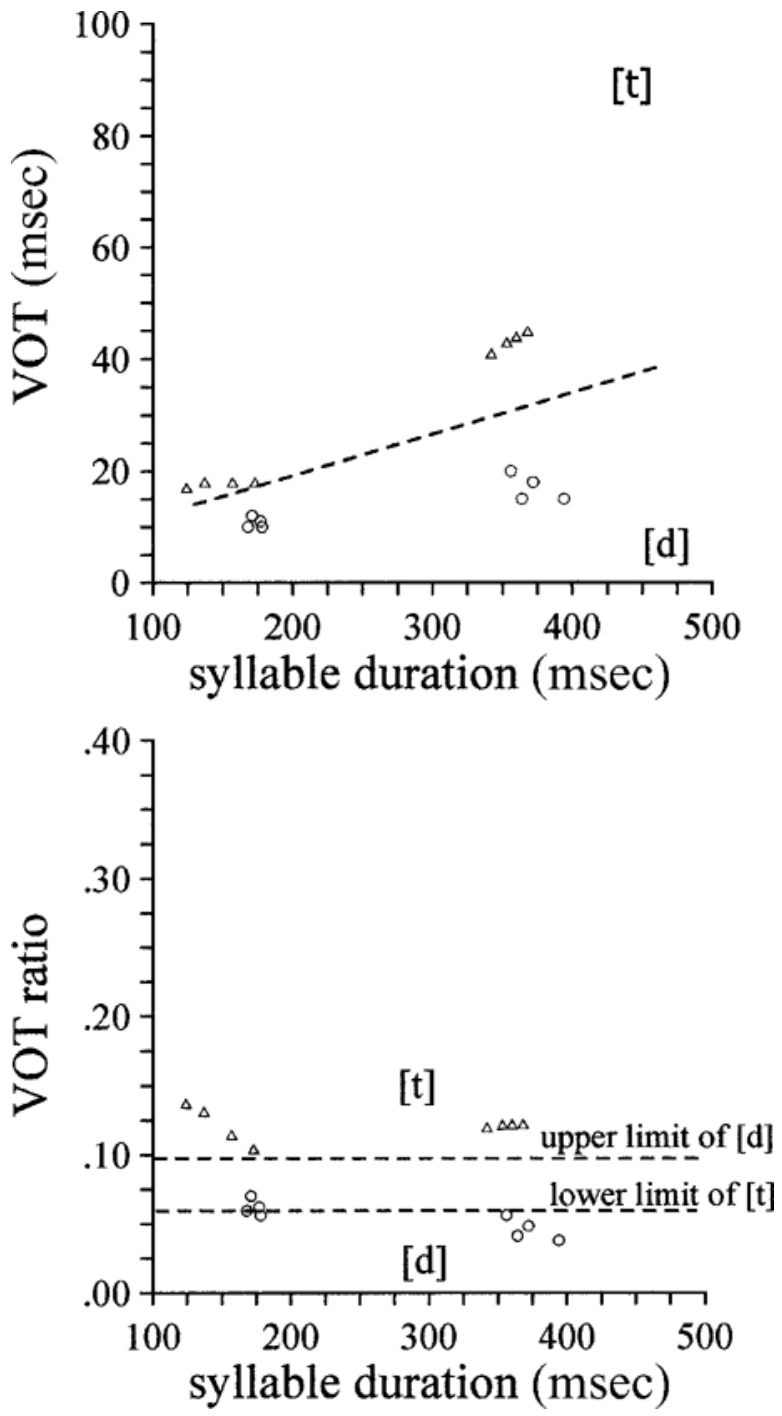

Figure 4. VOTs (top panel) and corresponding durational ratios of VOT (bottom panel) for selected syllables from Experiment 1 produced by different speakers. These syllables containing an initial $/ \mathrm{t} /$ or $/ \mathrm{d} /$ were used as stimuli in Experiment 2 . The dotted line in the top panel refers to an assumed midpoint boundary between best-fit regression lines, whereas the dotted lines in the bottom panel represent a boundary region specified by reference to the observed minimum ratio for $/ \mathrm{t} /$ and maximum ratio for /d / (see Figure 3).

were spliced from their utterance context at zero crossings of the waveform at points corresponding to the quasisilent gaps of the consonant closure (providing syllables heard as [dæp] or [tæp]). Each of the 16 syllables was first duplicated with a 2 -sec silence between the duplicates (a pair) and stored as a separate audio file. The files were then played back and recorded at two separate times on digital audiotape and in quasi-random order along with other pairs used in Experiment 3. The stimulus tape was constructed so that each trial consisted of a spoken identification number, a silence, two identical syllables (a pair) separated by a short silence, and then a longer silence (allowing for the judge's response) prior to another spoken number, and so on. The tape provided each of the 10 subjects two opportuni- ties to identify a syllable, giving a total of 20 identifications for each of the 16 syllables.

The test consisted of a forced-choice identification of the stimuli where the subjects listened to two presentations of a syllable prior to identifying, on a tally sheet, whether the heard context contained an initial $d$ or $t$. The subjects were provided with written instructions and listened to the stimuli in a sound-attenuating room through circumaural headphones (Beyerdynamic, Model DT 811). The stimulus tape was played back at a comfortable sound-pressure level with the use of the digital recording equipment mentioned in Experiment 1. Headphone output, estimated for 40 tokens by means of an IEC 318 coupler, a Brüel and Kjaer 4153 microphone, and a sonometer "F" setting, averaged $66.1 \mathrm{~dB}$ SPL. This level was maintained throughout Experiments 2 and 3.

\section{Results}

The 10 listeners correctly identified $100 \%$ of the tokens containing a syllable-initial/t/ $(80 / 80$ for each of the two speech rate conditions). However, scores for the stimuli containing an initial /d/ fell to 70\% (56/80) for syllables reflecting the fast speech condition and to $85 \%(68 / 80)$ for syllables representing the slow speech condition. A repeated measures comparison of the number of correct identifications across speaking rates for the stimuli containing /d/ showed that the differences were significant $[t(9)=2.71$, $p=.024]$. The results support the predictions above bearing on durational ratios but do not appear to reflect the distributions of absolute VOTs with respect to a shifting boundary as represented in Figure 4. That is, listeners' judgments suggest a degree of ambivalence for the stimuli containing/d/ for both speech rates, despite the large differences in absolute VOTs of $/ \mathrm{d} /$ and $/ \mathrm{t} /$ in the slow rate condition. However, this ambivalence in listeners' judgments appears to agree with the fact that ratios of /d/ VOT in the stimuli were near the boundary region (as is illustrated in the bottom panel of Figure 4). The results also show that listeners' judgments were highly accurate for tokens containing $/ \mathrm{t} /$. This fails to reflect the small millisecond differences between / $t$ / and /d/ VOTs in the fast speech condition but can be seen to conform to the fact that the ratios of / $/$ VOT were all outside the boundary region. Thus, the results show that there is a fluctuation in listeners' identifications when the durational ratios of VOT contained in the stimuli approach the criterion estimated from the production of $/ \mathrm{t} /$ and $/ \mathrm{d} /$, whereas judgments are highly accurate for ratios that are not in the boundary region. Experiment 3 was a further investigation of whether this boundary obtained through reference to the production data of Experiment 1 could be used to predict shifts in an identification function.

\section{EXPERIMENT 3}

\section{Method}

None of the speakers in Experiment 1 produced syllables containing / $\mathrm{t} / \mathrm{with}$ ratios of VOT smaller than .062 . Below this limit, only /d/ ratios occurred. If listeners rely on timing relations in speech in identifying voicing categories, a reduction of the duration of the syllable that increases the ratio of VOT above the .062 limit should create a shift in the identification function. The object of experi- 
ment 3 was to determine whether such changes in the durational ratios of VOT serve to predict listeners' identif ications when millisecond values of VOT and other attributes at vowel onsets are held constant. Attributes such as the duration and amplitude of release and $F 1$ transitions might influence the perception of syllable-initial stops and all of these might covary as a function of the degree of vocal-tract constriction in producing oral stops (Lisker, 1978; Lubker \& Parris, 1969; Summerfield \& Haggard, 1977). In considering these potential cues, the present experiment exploits a particular paradigm. Vowel portions of a test syllable with an initial /d/ were reduced in duration in order to increase the temporal ratio of events at vowel onsets. Millisecond values of VOT in such stimuli (as well as amplitude- and frequency-related information at vowel beginnings) were held constant, whereas the ratios of these events with respect to syllable duration varied in the direction of a/t/ category. This provided a means for observing the relative contribution or degree to which timing relations, as such, might influence listeners' judgments in the presence of other category-related information at vowel onsets.

Subjects, Stimuli, and Procedure. The stimuli used in this experiment were included in the audiotape presented to the 10 listeners of Experiment 2. These stimuli were constructed by splicing out portions of one of the syllables ([dæp]) associated with the slow speech condition of the preceding experiment. The syllable in question contained an initial /d/ that was correctly identified in all but 1 of the 20 presentations provided in Experiment 2. The original syllable context included a /d/ closure interval of $147 \mathrm{msec}$, a release duration of $20 \mathrm{msec}$, a VOT of $15 \mathrm{msec}$, and vocalic formants with durations of $227 \mathrm{msec}$. Editing functions of the aforementioned system (CSL) were used to delete, at zero crossings in the waveforms, midportions of the vowel segment so as to reduce the duration of the syllable from 175 to $75 \mathrm{msec}$ in 25 -msec steps $( \pm 3 \mathrm{msec}$ ). This procedure provided five stimuli with no perceptible clicks. The stimuli were arranged in quasi-random order on the audiotape along with the stimuli of the preceding experiment and their presentation followed the same procedure and format that has been described previously (see Experiment 2). That is, duplicates of the stimulus (a pair) provided the listener with two opportunities to hear the same stimuli prior to identifying on a tally sheet whether the initial consonant was $t$ or $d$. These pairs appeared at two different times on the audiotape so that a total of 20 identifications were performed for each of the five spliced syllables.

The original context presented a VOT ratio of .04, given to a syllable duration of $394 \mathrm{msec}$. To reach the estimated boundary ratio of .06 , a reduction of the syllable by $144 \mathrm{msec}$ was required. On this basis, the prediction was that a shift in the identification function would occur for reductions of $150 \mathrm{msec}$ and that, beyond this point, listeners' responses would favor the unvoiced category. Splicing was restricted by consideration of the maximal syllable rates observed in the first experiment (which conform to the reported average maximum for repeated CV syllables produced by male speakers in the subjects' age group; Kent, Kent, \& Rosenbek, 1987). That is, the reduction of $175 \mathrm{msec}$ provided a vowel segment of $50 \mathrm{msec}$, which was within the observed minimum vowel length of $40 \mathrm{msec}$ produced by the subjects.

\section{Results}

Figure 5 shows that, with the reduction in syllable duration, judges' identifications in terms of the voicing category of the initial consonant progressively shifted from $d$ to majority judgments of $t$. Reductions approaching $150 \mathrm{msec}$ correspond to chance-level judgments of $t(45 \%)$, marking the boundary point of the predicted shift in the identification function. A Cochran $Q$ test confirmed that the change in listeners' responses across the five reductions was significant $[Q(4)=17.692, p=.0014]$.
The reductions were restricted by consideration of the natural limits on speech rate and, even with a maximal reduction, the identification function did not shift to $100 \%$ $t$ judgments. This suggests that, aside from the timing of voice onsets, other details at vowel beginnings perhaps provided conflicting information on the category affiliation of the heard consonants. Nonetheless, the findings show that, with the millisecond values of events at vowel onsets being held constant, altering the timing ratio of VOT influenced listeners' identifications of the /d/ onset to the degree that listeners' judgments favored the voiceless category. The results also show that the boundary point that was inferred from the production data of Experiment 1 corresponds to a perceptual boundary, as suggested by the changes in the identification function.

\section{DISCUSSION AND CONCLUSION}

The results indicate that relative-time measures of VOT reveal distributions with stable boundaries across changes in speaking rates and that such boundaries can specify a perceptual criterion by which listeners judge the category affiliation of voice onsets. Both the perception of natural speech syllables (Experiment 2) and manipulated syllables (Experiment 3) confirm that the boundary defined with reference to limit values in the production of VOT ratios corresponds to a perceptual criterion. This must not be taken to imply that a perceptual boundary refers to a particular ratio that can generalize across contexts or languages.

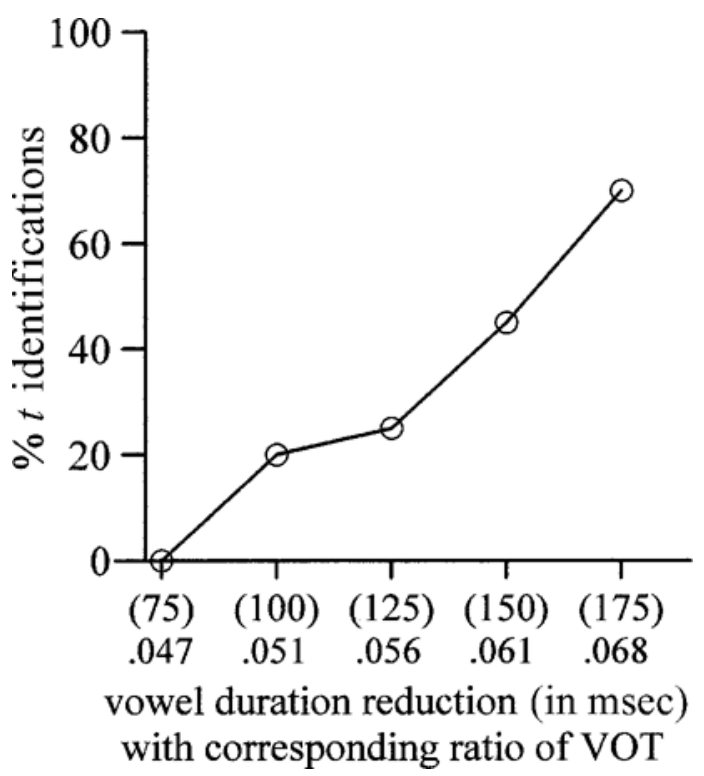

Figure 5. Percent identifications of $t$ for the syllable stimuli with varying reductions of vowel duration. The original syllable contained an initial / $\mathrm{d} /$ that was correctly identified in Experiment 2 in all but one of the 20 presentations. Note that the shift in the identification function that occurs for the syllable presenting a vowel reduction of $150 \mathrm{msec}$ corresponds to a ratio of VOT of .06, which is the boundary point for/t/ estimated from the production data of Experiment 1. 
Estimates of a criterion ratio obviously can vary in terms of the reference event - though all such measures could predict shifts in an identification function. That is, best-fit regression lines for $/ \mathrm{d} /$ and $/ \mathrm{t} /$ ratios of VOT manifested near-zero slopes across speaking rates regardless of whether the reference duration was $\mathrm{CV}, \mathrm{VC}$, or $\mathrm{CVC}$, and one could have inferred from these varying metrics different boundary ratios. Thus, the view is not that listeners refer to a specific ratio but that they attend to timing relations in speech that can encompass a number of events extending perhaps beyond the CV syllable.

The interpretation above and the present data stand in contrast to views expressed in recent reports on the perception of VOT. Kessinger and Blumstein (1998) have contended that, when VOT and vowel length vary inversely, listeners' identifications may be based more on changes in the following vowel than on the VOTs. This effect may not account for the results of Experiment 3. It will be recalled that the stimuli of that experiment involved a reduction in the duration of a spoken syllable containing an initial [d] and that this modification left the vowel onsets intact. The reductions created, to some extent, a shift in listeners' identifications toward a voiceless stop despite the presence of information at vowel beginnings relating to a voiced stop. Thus, it appears that listeners were attending to timing relations involving VOT and were not strictly influenced by vowel portions of the stimuli.

The preceding results also contrast with those of Utman (1998). The stimuli used in that study involved syllables where long voiceless VOTs were combined with short vowels and short voiceless VOTs with long vowel. Given that listeners identified the voiceless stop irrespective of vowel durations, the author concluded that timing relations did not affect listeners' responses. The stimuli used in the above Experiment 3 involved manipulations of vowel duration that conformed to observed durational ratios in speech (Experiment 1). In Utman, the "short" voiceless VOTs of the stimuli averaged $48 \mathrm{msec}$, whereas the "long" voiceless VOTs averaged $148 \mathrm{msec}$. With such onset times, creating a typical [d] VOT ratio of, say, .03 (see Figure 3) would have implied unusually long syllable durations of 1.6 and $4.9 \mathrm{sec}$. Utman's study thus referred to stimuli containing only large (voiceless) ratios of VOT. Such stimuli precluded the identification of voiced stops and did not provide a basis for invalidating an intrinsic timing hypothesis.

The present study constitutes the first demonstration that a boundary ratio derived from speech production data can correspond to a perceptual boundary, and this serves to clarify general claims in a number of reports to the effect that local timing relations can influence the identification of consonants (e.g., Pind, 1995; Port \& Dalby, 1982; Sawusch \& Newman, 2000; Summerfield, 1981). That is, the observations above support an intrinsic view of timing perception, though not in terms of earlier assumptions regarding the invariance issue. From the fact that temporal ratios in speech are not constant, intrinsic timing has been criticized in its failure to account for the perceptual constancy that is manifest in listeners' identifications of variable aspects such as VOT (see discussions by Pind, 1995, and Wayland et al., 1994). The present observations suggest that the perception of categories of VOT across speech rates need not imply measurable constants, or a process in which it is assumed that listeners actively shift a criterion value in identifying the heard VOTs. The results also bear some implications with respect to definitions of the invariance issue (Handel, 1984), where any attempt to define the physical basis of perceived categories or features is seen as a quest for invariance in speech (Lindblom, 1996). Timing ratios of voice onsets are, as Figure 3 shows, quite variable. However, a relative-time metric reveals distributions of VOT ratios with a stable boundary, and, as the results suggest, such patterns can intrinsically support listeners' identifications of variable VOTs in terms of voicing categories.

The previous interpretations are restricted to particular syllable-initial consonants and nonword contexts produced by English speakers. Several important issues are not addressed. Magloire and Green (1999) suggest that effects of changes in speaking rate on VOT can be restricted to languages that present relatively large (positive) VOTs. Furthermore, although the observations of VOTs in Experiment 1 are consistent with those reported in other studies (see the reference to Lane et al., 1995), measures taken from repeated syllables may not reflect the full range of values found in spontaneous speech. Finally, this study does not address the question of whether the effects of timing relations are strictly local or can imply rhythmical units extending beyond the syllable context, as suggested in certain early reports (e.g., Kozhevnikov \& Chistovich, 1965). A recent investigation by Sawusch and Newman (2000) taking into account issues of stimulus quality indicates that events occurring $250 \mathrm{msec}$ after a target consonant have no effect on listeners' responses (cf. Summerfield, 1981). Such a "temporal window" in perception approximates the average duration of syllables produced in conversational speech. What is less clear is how timing properties of preceding phrases can affect local timing relations and whether or not, across variable contexts, durational ratios maintain stable boundaries that support the perception of phonemic categories.

In conclusion, although the present results are restricted to particular utterances and involve a small group of speakers, the findings lend support to an intrinsic view of timing perception. The relevance of this view should not be taken to rest on the finding of invariant timing relations in speech. The observations show that, despite the variability of durational ratios of VOT across speaking rates, such ratios present distributions for voiced and voiceless stops with stable boundaries that can intrinsically specify a perceptual criterion according to which listeners identify voicing categories. Further work is needed to verify whether or not timing relations can determine a stable perceptual boundary for voicing attributes in varying contexts and 
languages. One must also clarify how the properties of precursor phrases or sentences affect the perception of local timing relations in test syllables. It is unclear whether such effects require assumptions of extrinsic normalization or whether the effects can be intrinsically specified by timing relations extending to larger rhythmic units.

\section{REFERENCES}

Abramson, A. S., \& LisKer, L. (1970). Discriminability along the voicing continuum: Cross-language tests. In Proceedings of the 6th International Congress of Phonetic Sciences (pp. 569-573). Prague: Academia.

Boucher, V. (1988). A parameter of syllabification for VstopV and relative-timing invariance. Journal of Phonetics, 16, 299-326.

Brown, W., Morris, R , \& WeIss, R. (1993). Comparative methods for measurement of VOT. Journal of Phonetics, 21, 329-336.

Clarkson, R. L., Eimas, P. D., \& Marean, G. C. (1989). Speech perception in children with histories of otitis media. Journal of the Acoustical Society of America, 85, 926-933.

DieHL, R. L., \& WALSH, M. A. (1989). An auditory basis for the stimuluslength effect in the perception of stops and glides. Journal of the Acoustical Society of America, 85, 2154-2164.

Dupoux, E., \& Green, K. P. (1997). Perceptual adjustment to highly compressed speech of talker and rate changes. Journal of Experimental Psychology: Human Perception \& Performance, 23, 914-928.

Eimas, P. D., Miller, J. L., \& Jusczy K, P. W. (1987). On infant perception and the acquisition of language. In S. Harnard (Ed.), Categorical perception: The groundwork of cognition (pp. 161-195). New York: Cambridge University Press.

Fowler, C. A. (1980). Coarticulation and theories of extrinsic timing. Journal of Phonetics, 8, 113-133.

Green, K. P., Stevens, E. B., \& Kuhl, P. K. (1994). Talker continuity and the use of rate information during phonetic perception. Perception \& Psychophysics, 55, 249-260.

HANDEL, S. (1984). Listening: An introduction to the perception of auditory events. Cambridge, MA: MIT Press.

Harris, K., Tuller, B., \& Kelso, J. A. S. (1986). Temporal invariance in the production of speech. In J. S. Perkell \& D. H. Klatt (Eds.), Invariance and variability in speech processes (pp. 243-252). Hillsdale, NJ: Erlbaum.

KELLER, E. (1987). The variation of absolute and relative measures of speech activity. Journal of Phonetics, 15, 335-347.

KELler, E. (1989). Predictors of subsyllabic durations in speech motor control. Journal of the Acoustical Society of America, 33, 322-326.

Kelso, J. A. S., Saltzman, E, \& Tuller, B. (1986). The dynamical perspective on speech production: Data and theory. Journal of Phonetics, 14, 129-159.

Kelso, J. A. S., \& Tuller, B. (1985). Intrinsic time in speech production: Theory, methodology, and preliminary observations (Haskins Laboratories Status Report on Speech Research SR-81, pp. 23-39). New Haven, CT: Haskins Laboratories.

Kent, R. D., Kent, J. F., \& Rosenbek, J. C. (1987). Maximum performance measures of speech production. Journal of Speech \& Hearing Research, 52, 367-387.

Kessinger, R. H., \& Blumstein, S. E. (1998). Effects of speaking rate on voice-onset time and vowel production: Some implications for perception studies. Journal of Phonetics, 26, 117-128.

KIDD, G. R. (1989). Articulatory-rate context effects in phoneme identification. Journal of Experimental Psychology: Human Perception \& Performance, 15, 736-748.

Kleinbaum, D., Kupper, L., \& Muller, K. (1988). Applied regression analysis and other multivariate methods. Boston: PWS-Kent.

Kozhevnikov, V., \& Chistovich, L. (1965). Speech: Articulation and language. Washington: Clearinghouse for Federal Technical and Scientific Information.

Kuhl, P. K., \& Miller, J. D. (1978). Speech perception by the chinchilla: Identification functions for synthetic VOT stimuli. Journal of the Acoustical Society of America, 63, 905-917.

Lane, H., Wozniak, J., Matthies, M., Svirsky, M., \& Perkell, J. S.
(1995). Phonemic resetting versus postural adjustments in the speech of cochlear implant users: An exploration of voice-onset time. Journal of the Acoustical Society of America, 98, 3096-3106.

LindBLOM, B. (1996). Role of articulation in speech perception: Clues from production. Journal of the Acoustical Society of America, 99, 1684-1691.

LISKER, L. (1978). Rapid vs. rabid: A catalogue of acoustic features that may cue the distinction (Haskins Laboratories Status Report on Speech Research, SR-54, pp. 127-132). New Haven, CT: Haskins Laboratories.

LÖFQVIST, A. (1991). Proportional timing in speech motor control. Journal of Phonetics, 19, 343-350.

Lubker, J., \& PARRIS, P. (1969). Simultaneous measurements of intraoral pressure, force of labial contact, and labial electromyographic activity during production of the stop consonant cognates $/ \mathrm{p} /$ and $/ \mathrm{b} /$. Journal of the Acoustical Society of America, 47, 625-633.

Luce, P., \& Charles-Luce, J. (1985). Contextual effects on vowel duration, closure duration, and the consonant/vowel ratio in speech production. Journal of the Acoustical Society of America, 78, 1949-1957.

Magloire, J., \& GreEn, K. P. (1999). A cross-language comparison of speaking rate effects on the production of voice onset time in English and Spanish. Phonetica, 56, 158-185.

Miller, J. L., Aibel, I. L., \& Green, K. (1984). On the nature of ratedependent processing during phonetic perception. Perception \& Psychophysics, 35, 5-15.

Miller, J. L., \& BAER, T. (1983). Some effects of speaking rate on the production of $/ \mathrm{b} /$ and /w/. Journal of the Acoustical Society of America, 73, 1751-1755.

Miller, J. L., Green, K. P., \& Reeves, A. (1986). Speaking rate and segments: A look at the relation between speech production and speech perception for the voicing contrast. Phonetica, 43, 106-115.

Miller, J. L., \& Liberman, A. M. (1979). Some effects of later-occurring information on the perception of stop consonant and semivowel. Perception \& Psychophysics, 25, 457-465.

Miller, J. L., \& Volaitis, L. E. (1989). Effect of speaking rate on the perceptual structure of a phonetic category. Perception \& Psychophysics, 46, 505-512.

Nittrouer, S., Munhall, K., Kelso, J. A. S., Tuller, B., \& HarRIS, K. (1988). Patterns of interarticulator phasing and their relation to linguistic structure. Journal of the Acoustical Society of America, 84, 1653-1661.

PIND, J. (1995). Speaking rate, voice-onset time, and quantity: The search for higher-order invariants for two Icelandic speech cues. Perception \& Psychophysics, 57, 291-304.

Pisoni, D. B. (1986). Contextual variability and the problem of acoustic-phonetic invariance in speech. In J. S. Perkell \& D. H. Klatt (Eds.), Invariance and variability in speech processes (pp. 155-161). Hillsdale, NJ: Erlbaum.

Pisoni, D. B., Carrell, T. D., \& Gans, S. J. (1983). Perception of the duration of rapid spectrum changes in speech and nonspeech signals. Perception \& Psychophysics, 34, 314-322.

PORT, R. F. (1981). Linguistic timing factors in combination. Journal of Acoustical Society of America, 69, 262-274.

PorT, R. F., \& DALBY, J. (1982). Consonant/vowel ratio as a cue for voicing in English. Perception \& Psychophysics, 32, 141-152.

Port, R. F., \& RotunNo, R. (1979). Relation between voice-onset time and vowel duration. Journal of the Acoustical Society of America, 66, 654-662.

Sawusch, J. R., \& Newman, R. S. (2000). Perceptual normalization for speaking rate II: Effects of signal discontinuities. Perception \& Psychophysics, 62, 285-300.

SineX, D., \& NARAY AN, S. (1994). Auditory-nerve fiber representation of temporal cues to voicing in word-medial stop consonants. Journal of the Acoustical Society of America, 95, 897-903.

StARK, R, \& TAllal, P. (1988). Language, speech, and reading disorders in children: Neuropsychological studies. Boston: College-Hill.

Summerfield, A. Q. (1975). How a full account of segmental perception depends on prosody and vice versa. In A. Cohen \& S. Nooteboom (Eds.), Structure and process in speech perception (pp. 51-68). New York: Springer-Verlag.

Summerfield, [A.] Q. (1981). On the articulatory rate and perceptual 
constancy in phonetic perception. Journal of Experimental Psychology: Human Perception \& Performance, 7, 1074-1095.

Summerfield, [A.] Q., \& HaGgard, M. (1977). On the dissociation of spectral and temporal cues to the voicing distinction in initial stop consonants. Journal of the Acoustical Society of America, 62, 435-448.

Tuller, B., \& Kelso, J. A. S. (1984). The timing of articulatory gestures: Evidence for relational invariants. Journal of the Acoustical Society of America, 76, 1030-1036.

Tuller, B., \& Kelso, J. A. S. (1991). The production and perception of syllable structure. Journal of Speech \& Hearing Research, 34, 501508.

UTMAN, J. A. (1998). Effects of local speaking rate context on the per- ception of voice-onset timing in initial stop consonants. Journal of the Acoustical Society of America, 103, 1640-1653.

Volaitis, L. E., \& Miller, J. L. (1992). Phonetic prototypes: Influence of place of articulation and speaking rate on the internal structure of voicing categories. Journal of the Acoustical Society of America, 92, 723-735.

Way land, S. C., Miller, J. L., \& Volaitis, L. E. (1994). The influence of sentential speaking rate on the internal structure of phonetic categories. Journal of the Acoustical Society of America, 89, 2694-2701.

(Manuscript received November 1, 1999; revision accepted for publication March 20, 2001.) 